\title{
Wire-track infections in ilizarov technique how to manage that?
}

\begin{abstract}
Wire-track infection is very common in Ilizarov fixation. We can follow a commonly accepted some steps to reduce the occurrence of infection. Improvement of technique can make wire-track infection less. Preoperative planning, meticulous intelligent surgical technique, patients understanding regarding the apparatus, and regular patient monitoring all these can minimize the wire-track infections.
\end{abstract}

Keywords: Ilizarov fixation, Wire-track, Infection
Volume 9 Issue I - 2017

\author{
Bari MM, ${ }^{\prime 2}$ Islam Shahidul, ${ }^{2}$ Shetu $\mathrm{NH}^{2}$ \\ Mahfuzer RM ${ }^{2}$ \\ 'Consultant, Bari-llizarov Orthopaedic Centre, Bangladesh \\ ${ }^{2}$ Prof. Ph.D, Chief Consultant, Bari-llizarov Orthopaedic Centre, \\ Visiting and Honored Prof., Russian llizarov Scientific Centre, \\ Russia
}

\begin{abstract}
Correspondence: Bari MM, Chief Consultant, Bari-llizarov Orthopaedic Centre,Visiting and Honored Professor Russian llizarov Scientific Centre, Kurgan, Russia, Tel 8800000000000
\end{abstract} Email bari.ilizarov3I@gmail.com

Received: September II, 2017 | Published: September 19,

2017

\section{Introduction}

Wire-track infection is common in Ilizarov fixation. Any wire that violates the skin barrier is at risk for developing a wire-track infection. Iobst and Liu reviewed the external fixation literature from 1980 to 2014 and found $27.4 \%$ risk of wire-track infection. ${ }^{1}$

\section{Literature Review}

There is no uniform definition of what constitutes a wire-track infection. The following variables have been used to diagnose a wiretrack infection: erythema, purulent drainage, response to antibiotics or requiring the removal of offending wire. There is no universally accepted system that satisfies all surgeons. None of the classification systems has been empirically developed or tested for their reliability.

There is not a standard method of diagnosing wire-track infection in the literature. If a wire-track infection develops at any point during the course of treatment, then the patient is thought to be infected. The drawback of this method is that it does not differentiate between infection at a single wire site or multiple wire sites within one patient. The other common method of observing wire-track infection is per wire site..$^{2-4}$

Finally there is not even agreement among Ilizarovians regarding what to call wire-track infections. According to dictionary.com, the definition of a track is a course or route followed. The definition of a track is an expanse or area of land, water etc. There are studies attempting to determine the optimal cleansing solution, the most effective dressing type, or the ideal frequency of cleaning..$^{5-8}$ Even a single patient's Ilizarov frame, not all the wires are equally at risk for developing an infection (Figure 1).

Each wire in a particular Ilizarov fixator should be considered an individual entity whose wire-bone and wire-skin interface health is determined by a host of factors that begin the moment the wire-skin barrier is breached in the operation theatre.

\section{Some tips and tricks to reduce wire-track infections}

Preoperative assessment of patients soft tissue envelope is absolutely mandatory. A plan for wire placement may be performed to avoid areas of open wounds muscle flaps or the zone of injury. Evaluation should be done for quality of bone. ${ }^{8}$

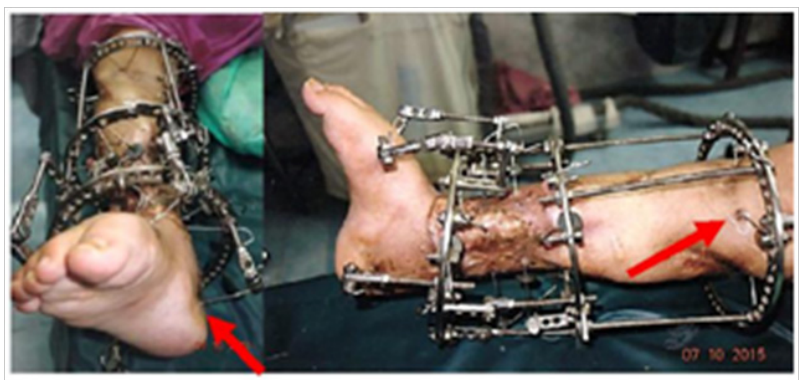

Figure I llizarov fixator patient's tibia showing common scenario in which wires may cause risks of infection.

Stress on the wire bone interface can be reduced using the accurate size of Ilizarov rings and building a very stable Ilizarov frame construct. We must devise a plan for number and position of wires to be inserted. A unstable Ilizarov frame construct will increase the stress at each individual wire bone interface which can lead to loosening and inflammation. ${ }^{4,8}$ Our goal is to introduce a traumatic meticulous insertion of the wire through the skin, soft tissue and bone. It is absolutely mandatory to manipulate the limb through a full range of motion at the end of the surgery to identify and release any area of skin tension. ${ }^{8}$

Skin and muscle motion over any wire should be minimized by special dressing techniques. For thin wire fixators rubber stopper is put over the gauge to apply mild pressure on the skin. Excessive pressure should be avoided. All wire care should include daily sterilization with an antiseptic, like rectified sprit or chlorhexidine gluconate.

At the first sign of wire track infection, broad spectrum antibiotics should be given, local wire care should be intensified and the wire site should be incised to promote draining if necessary. If the infection does not improve with these measures, the wire may have to be removed. A replacement olive wire should be inserted. With Ilizarov apparatus, this is relatively simple: a olive wire or smooth wire can be placed to a nearly hole or dropped off the ring on a post to avoid the infected wire site. Wires should be inserted using a gauge soaked in 
alcohol or rectified sprit to cool and disinfect the wire during drilling (Figure 2).

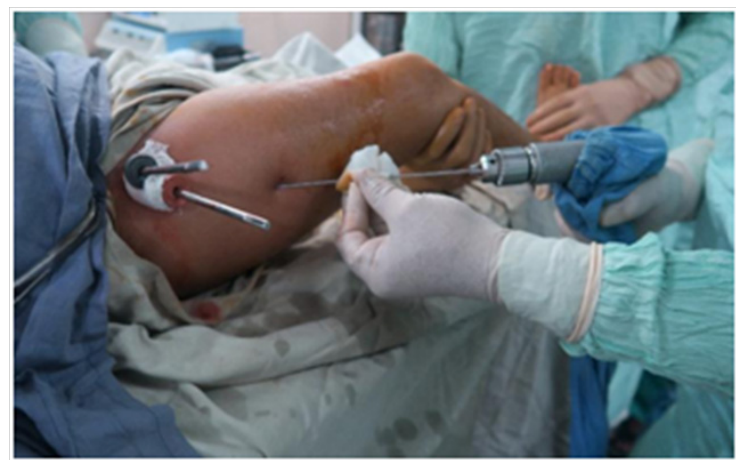

Figure 2 Shows how to hold the wire with soaked gauge during treatment.

The wire should be pushed through the soft tissues to the cortex and then dilled through the soft tissues after penetrating the $2 \mathrm{nd}$ cortex. We are using half pins only in the proximal and upper $1 / 3$ rd of femur. Half pins should be predrilled and inserted by hand.

We are always following the original Kurgan principles regarding wire insertion to avoid heat

1. Avoid cannulated drills wherever possible.

2. Low-speed drilling rather than continuous drilling on high speed.

3. Continuous irrigating while drilling wire spirit.

4. Avoid unicortical half pin placement. ${ }^{4,8}$

The wire site should be irrigated after insertion to remove any bony particles in the surrounding soft tissue. Pin sites should be covered and compressed by rubber stopper with sterile dressing after insertion to absorb any blood. The dressing well prevents skin tenting or haematoma formation. ${ }^{4}$
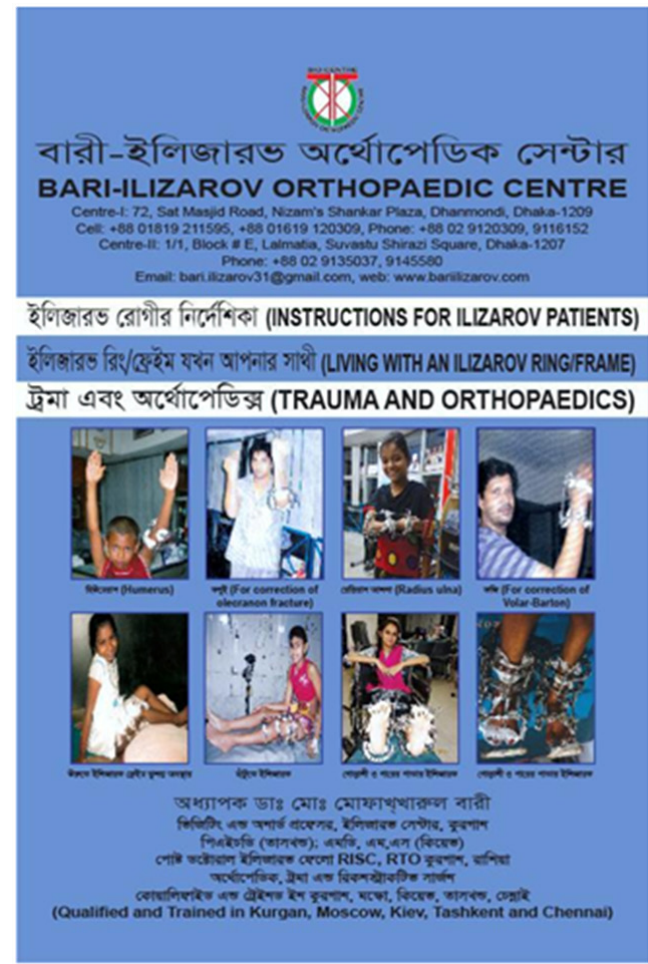

Figure 3 Instructions for llizarov Patients (www.bariilizarov.com).
Some wire locations such as periarticular region, have increased soft tissue movement around the wire which can lead to irritation at the site. Stabilizing the wire and skin interface by rubber stopper with sterile gauge will limit the micro motion at these sites. As a result we can minimize the wire-track infection. ${ }^{4}$

Patient education is another critical element to decreasing the risk of wire-track infection. We have written a small guideline for the patient. The patient should also be reminded to keep the limb elevated at all times except for range of motion exercise or walking (Figure 3).

Elevation will reduce aedema around the wires8. It is important for the surgeon to maintain close observation of the patient with frequent follow-up at least weekly monitoring the wire site by the Ilizarov surgeon.

\section{Conclusion}

Meticulous preoperative planning and insertion technique are mandatory. Wire design will help to decrease the risk of wire-track infection. Preoperative planning, meticulously surgical technique, patient understands regarding the Ilizarov frame and close monitoring all these are very critical to minimizing the wire site infections.

\section{Acknowledgments}

None.

\section{Conflicts of interest}

None.

\section{References}

1. Iobst CA, Liu RW Systematic review of incidence of pin track infections associated with external fixation. $J$ Limb Lengthen Reconstr. 2016;2(1):6-16.

2. Ilizarov GA The principles of Ilizarov method. Bull Hosp. Jt Dis Orthop Inst. 1988;48:1-11.

3. Paley D Problems, obstacles, and complications of limb lengthening by the Ilizarov technique. Clin Orthop Relat Res. 1990;250:81-104.

4. MM Bari A color atlas of limb lengthening surgical reconstruction and deformity correction by Ilizarov technique. p. 2013;42-43.

5. Henry C Pin Sites: Do we need to clean them? Pract Nurs. 1996;7(4):12-17.

6. Egol KA, Paksima N, Puopolo S et al. Treatment of external fixation pins about the wrist: A prospective, randomized trial. J Bone Joint Surg Am. 2006;88(2):349-354.

7. Grant $\mathrm{S}$, Kerr D, Wallis M, Pitchford D Comparison of povidone-iodine solution and soft white paraffin ointment in the management of skeletal pin-sites: A pilot study. J Orthop Nurs. 2005;9(4):218-225.

8. Cavusoglu AT, Er MS, Inal $\mathrm{S}$ et al. Pin site care during circular external fixation using two different protocols. $J$ Orthop Trauma. 2009;23(10):724-730 